\title{
Payer Perspectives on Intravenous versus Subcutaneous Administration of Drugs
}

\section{Robert S Epstein (1D) \\ Epstein Health, LLC, Woodcliff Lake,} NJ, USA
Correspondence: Robert S Epstein Epstein Health, LLC, 50 Tice Boulevard, Suite 300, Woodcliff Lake, NJ, 07677, USA

Tel + I 20I-285-5800, ext. 102

Email repstein@epsteinhealth.com

\begin{abstract}
The coronavirus disease 2019 (COVID-19) pandemic has brought increased attention to vulnerable populations such as older or immunocompromised patients and heightened the focus on alternatives to intravenous (IV) formulations, particularly those that may be administered in a non-clinical setting. Among these alternative formulations are subcutaneous (SC) injections, which comprise an increasing share of commercialized and pipeline therapies. While much has been published about the benefits and limitations of IV versus SC administration to patients and health systems, less attention has been given to payer considerations regarding these routes of administration. Accordingly, this article provides payer perspectives on some of the key differences between IV and SC administration as they relate to management and billing, cost, treatment adherence and safety, and patient preference and quality of life. The benefits and limitations of these drug administration routes to key healthcare stakeholders - namely patients, physicians, and payers - are also discussed. Considerations of relevance are highlighted, including the potential for misalignment of stakeholder interests and countervailing factors that may impact decisionmaking about IV and SC formulations.
\end{abstract}

Keywords: drug utilization management, formularies, pharmacy benefits management

\section{Introduction}

The coronavirus disease 2019 (COVID-19) pandemic has heightened the sensitivity of patients, healthcare professionals (HCPs), and payers to outpatient intravenous (IV) infusion treatments due to concerns about exposing vulnerable patients to serious infection. Organizations such as The National Home Infusion Association have suggested that patients use alternative routes of administration, including intramuscular or subcutaneous (SC) injections, where clinically appropriate. ${ }^{1}$ In the context of cancer care, it has been advised that HCPs consider alternative treatments to infusions, including the use of oral cancer therapies, with telehealth visits where appropriate in order to minimize the threat of contracting COVID- $19 .^{2}$

There are many other reasons beyond the COVID-19 pandemic for payers to consider the growing injectable space and alternative management strategies where clinically appropriate. In recent years, there has been a noticeable increase in the number of injectable drugs in the marketplace. ${ }^{3}$ A recent evaluation of the global research and development pipeline by delivery route in 2019 revealed that $55 \%$ of all products being studied for pre-regulatory approval are injectable drugs. ${ }^{4}$ Additionally, an analysis of 2018 US prescription sales data from the IQVIA National Sales Perspective Database showed that the top three drugs by expenditure were injectables (adalimumab, insulin glargine, and etanercept) and 
accounted for $\$ 36.4$ billion of the $\$ 476.2$ billion in total drug expenditure. ${ }^{5}$ In the injectables space, there has also been an increasing trend towards SC delivery of therapeutic proteins versus IV administration ${ }^{6}$ (Figure 1). Although much has been published about the benefits and limitations of IV versus SC administration to patients and health systems, less attention has been given to payer considerations regarding these routes of administration. The purpose of this article is to provide payer perspectives on some of the key differences between IV and SC administration; highlight some of the key benefits and limitations of these formulations to patients, physicians, and payers; and propose considerations of relevance to payers.

\section{Coding, Management, and Billing Implications of IV vs SC Formulations} In the United States, IV and SC drugs are differently coded, adjudicated, managed, and billed; these differences have material implications for payers. Most drugs that are administered via the SC route (particularly those that are selfadministered) fall under the pharmacy benefit management (PBM) system, which permits payers to have complete visibility into: (1) the labeler (manufacturer/distributor); (2) the specific product strength, dosage form, and formulation; and, (3) the trade package size and types via the assigned
National Drug Code (NDC), a unique 10-digit, 3-segment number assigned to a SC product upon approval by the US Food and Drug Administration (FDA) (Table 1). ${ }^{7,8}$ The transparency of NDC billing enables easier adjudication and time-sensitive management of claims and also allows for more accurate billing and better cost management.

IV-administered drugs generally fall under the health plan management system and are coded using the Healthcare Common Procedure Coding System (HCPCS) (Table 1). ${ }^{8,9}$ HCPCS codes are not as specific and transparent as NDC codes; are updated on a quarterly basis (in contrast to NDC codes which are updated monthly); and have delayed timing of data availability post-drug launch, which may impact the timeliness of claims submissions (Table 1$){ }^{7}$ Coding for IV-administered drugs is more complex as it may require additional reporting of associated services and the application of a complex series of rules to define the total billable infusion time. Given these complexities, some payers prefer to manage their utilization of injectable therapies whenever possible using the PBM system, where formulary adherence, prior authorization in real-time, step therapy mandates, patient copayment, and a variety of other management techniques are more easily and quickly achieved. Payers, including United Healthcare, are increasingly mandating or requesting that the NDC billing format be applied to all physician claims, although this is not universal. ${ }^{7}$

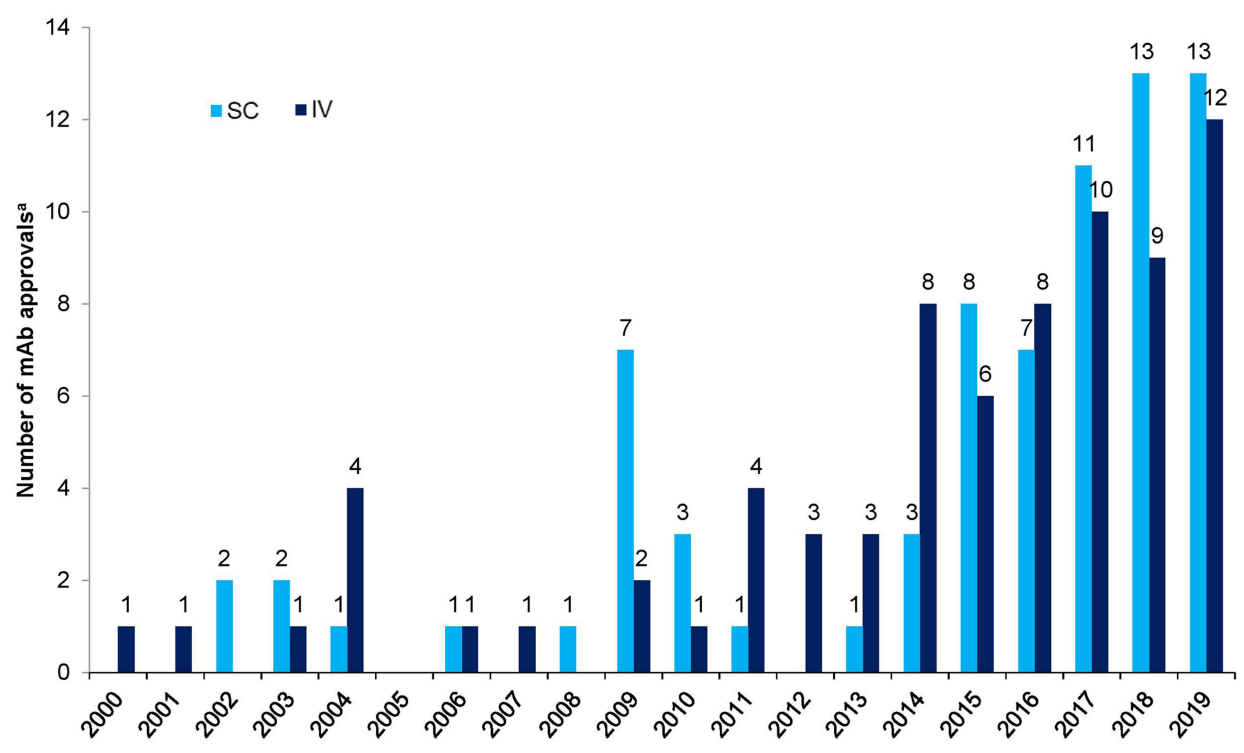

Figure I Subcutaneous versus intravenous monoclonal antibody approvals in the United States from 2000 to $2019 .{ }^{\text {a}}$ Figure classification counts different formulations and combination devices for a given mAb as separate product approvals. Adapted from Adv Drug Deliv Rev, Sanchez-Felix M, Burke M, Chen HH, Patterson C, Mittal S. Predicting bioavailability of monoclonal antibodies after subcutaneous administration: Open innovation challenge. Copyright (2020), with permission from Elsevier. ${ }^{6}$

Abbreviations: IV, intravenous; mAb, monoclonal antibody; SC, subcutaneous. 
Table I Some of the Key Differences in Coding, Adjudication, Management, and Billing Between IV and SC Administered Drugs

\begin{tabular}{|l|l|l|}
\hline Features & IV Administration & $\begin{array}{l}\text { SC } \\
\text { Administration }\end{array}$ \\
\hline Coding & HCPCS codes & NDC codes \\
\hline $\begin{array}{l}\text { Specificity of } \\
\text { coding }\end{array}$ & Non-specific & Specific \\
\hline $\begin{array}{l}\text { Timing of data } \\
\text { availability }\end{array}$ & Delayed & Immediate \\
\hline Visibility & Often not transparent & Fully transparent \\
\hline Setting & $\begin{array}{l}\text { Office/outpatient hospital - } \\
\text { extra costs and codes }\end{array}$ & $\begin{array}{l}\text { Home - no } \\
\text { additional costs }\end{array}$ \\
\hline $\begin{array}{l}\text { Provider } \\
\text { services/costs }\end{array}$ & Nurse/MD injection codes & $\begin{array}{l}\text { None if self- } \\
\text { injectable }\end{array}$ \\
\hline
\end{tabular}

Abbreviations: HCPCS, Healthcare Common Procedure Coding System; IV, intravenous; NDC, National Drug Code; SC, subcutaneous.

\section{Cost Implications of IV vs SC Formulations}

Transitioning from IV to SC administration can offer distinct cost advantages to payers. Firstly, in contrast to IV infusions, many SC medications (eg, rituximab and belimumab) ${ }^{10,11}$ do not require premedication, resulting in direct cost savings. For example, an analysis of the cost of IV infusion therapy for rheumatoid arthritis delivered in a hospital-based infusion center estimated premedication costs per infusion at $\$ 7$ and $\$ 19$ (2017 US dollars) for infliximab and rituximab, respectively. ${ }^{12}$ When applied to a large patient volume, premedication can have a considerable impact on the cost of care. Moreover, in a claims analysis of the Medco Health Solutions PBM database comprising 1090 US patients treated with biologics for rheumatoid arthritis (SC-administered etanercept and adalimumab, and IV-administered infliximab and abatacept), the cost per effectively-treated patient was approximately $\$ 16,000$ less with the SC medications versus IV. ${ }^{13}$ Likewise, an evaluation of the budget impact of the introduction of SC rituximab to US health plans found that switching cancer patients from IV to SC rituximab reduced total pharmacy and administration costs by $\$ 223,000$ for commercial health plans in the year with the highest conversion rate. ${ }^{14}$ Similar findings with oncology biologics have been reported across countries and study centers despite differences in healthcare systems and payer types. ${ }^{15-18}$

Examining indirect costs along with direct costs is another important consideration for some payers when comparing IV versus SC delivery. In a study of HER2positive breast cancer patients in Sweden, the overall societal cost (a combination of direct medical, direct nonmedical and indirect costs) of IV trastuzumab was higher than that of hospital-based SC delivery. ${ }^{19}$ More patients receiving IV treatment took time off from work compared to those on the SC regimen ( $14 \%$ vs $5 \% ; p=0.0223)$. Patient time spent at the hospital was significantly greater with IV than SC administration for initial (101 minutes longer) and subsequent (23 minutes longer) treatments with trastuzumab. Indirect costs arising from production loss and lost leisure time were also higher with IV versus SC delivery for initial ( $€ 140$ higher) and subsequent ( $€ 16$ higher) trastuzumab treatments. A pharmacoeconomic analysis of patients in Italy who initially received IV rituximab showed that at-home administration of SC rituximab led to fewer working days lost by patients and caregivers and a marked reduction in travel costs, resulting in a $70 \%$ decrease in indirect costs with no compromise to safety or adherence. ${ }^{20}$

\section{Treatment Adherence and Safety with IV vs SC Formulations}

Out of concern about treatment adherence and managing adverse side effects, some payers may prefer IV administration in a healthcare facility over SC delivery in a nonclinical setting as the former allows for direct observation by HCPs that the patient received the drug as prescribed and enables a prompt response to any adverse events while the patient is present. It should however be noted that SC therapies delivered by newer technologies, such as Bluetooth-enabled wearable devices, enable HCPs to assess and improve adherence in real-time. For instance, the Rebismart ${ }^{\mathbb{B}}$ autoinjector (Merck KGaA, Darmstadt, Germany) can send injection reminders to patients, log drug administration, and export and analyze data through its companion Mitra ${ }^{\circledR}$ computer application (Merck Serono, Darmstadt, Germany). ${ }^{21,22}$ Such devices may be leveraged for continuous monitoring and personalized education and messaging - all of which can improve patient adherence, safety, and outcomes — and can be utilized in conjunction with specialty pharmacy practices such as outbound calls and targeted messages. The recent changes in physician coding and billing for remote patient monitoring and telehealth incentivize this as well. ${ }^{23}$

SC delivery mechanisms such as autoinjectors have also been found to reduce patients' anxiety about self- 
administered injections, potentially improving treatment adherence and satisfaction. ${ }^{24} \mathrm{~A}$ study of patients with multiple sclerosis receiving interferon beta-1a via $\mathrm{SC}$ injection with Rebismart ${ }^{\circledR}$, which has adjustable settings for injection speed and depth of needle insertion, ${ }^{25}$ reported a median adherence of $96.5 \%$ over the 5 -year study period. ${ }^{21}$ This increased adherence was associated with a lower risk of disease relapse. Among multiple sclerosis patients receiving disease-modifying therapies, autoinjector use has been reported as the strongest predictor of treatment adherence at 24 months. ${ }^{25}$

Importantly, SC administration has been shown to be safe and well-tolerated. For example, in a review of 63 publications investigating the safety of IV and SC biologics approved for cancer treatment, the proportion of studies demonstrating fewer adverse events with SC versus IV was slightly higher than those showing no statistically significant difference. Only two trastuzumab-related studies were found to favor IV administration. ${ }^{16}$ Moreover, evidence suggests that SC administration may be associated with an improved safety profile in terms of reduced infusion-related reactions compared to IV infusions. ${ }^{26-28}$ Other methods of venous access such as indwelling catheters have been associated with complications such as infection, migration/malpositioning, bleeding, and thrombosis. ${ }^{29}$ Given these complications, the feasibility of SC injection as a viable alternative to venous catheterization may be worthy of investigation.

\section{Patient Preference and Quality of Life with IV and SC Formulations}

Given their implications for patient satisfaction and treatment adherence, patient quality of life and preference should be an important consideration when making formulary decisions. ${ }^{20} \mathrm{~A}$ systematic rapid evidence assessment of the humanistic impact of SC and IV formulations of oncology therapies showed that patients had a clear preference for SC administration and reported better health-related quality of life. ${ }^{16}$ In a cross-sectional study that switched 43 patients with systemic lupus erythematosus from IV or SC (pre-filled syringe) belimumab to selfadministered SC doses via autoinjector, all 21 interviewed patients found the autoinjector convenient. ${ }^{30}$ Of these, $81 \%$ reported a positive experience using the autoinjector. ${ }^{30}$ Questionnaire responses showed that $76 \%$ (32/42) of patients who switched from IV belimumab preferred the autoinjector based on convenience, cost, time savings, and decreased injection pain. ${ }^{30}$ Autoinjector use also improved daily function as well as the ability to work compared with IV administration. ${ }^{30}$ Likewise, in a systematic review of randomized controlled trials and crossover studies that evaluated patient preference for SC versus IV formulations, four out of six studies reported a patient preference for $\mathrm{SC}$ administration. ${ }^{31}$ The primary factors underlying this preference were the convenience of in-home treatment and the associated time savings. ${ }^{31}$ These benefits extend to caregivers as well, with SC dosing alleviating their care burden ${ }^{32}$ and reducing work absences. ${ }^{20}$ Despite the aforementioned benefits of SC administration, it should be recognized that IV administration may be preferred by some patients as it facilitates inperson access to and interaction with HCPs in a clinical setting. ${ }^{32}$

\section{Countervailing Considerations}

For payers, managing IV and SC treatment options often involves complex decision-making, as can be illustrated using the example of human immunoglobulins. Immunoglobulins are among the most complex specialty drugs for payers to manage due to the large number of products currently on the market, each with varying doses, formulations, and indications; the prescription of off-label uses; and the differing safety and tolerability of IV (IVIG) and SC immunoglobulin (SCIG) formulations based on patient characteristics and route of administration. ${ }^{33,34}$ Shifting the site of care to the home setting and switching patients from IVIG to SCIG has been shown to be efficacious and cost-saving, and surveys found that patients with primary immunodeficiencies generally prefer in-home immunoglobulin administration. ${ }^{33}$ This notwithstanding, immunoglobulin infusions in a clinical setting may still be preferred for some patients, such as those at risk of adverse events and those who are non-adherent to therapy. ${ }^{35}$ Immunoglobulins underscore the importance of keeping an open formulary that affords HCPs the flexibility to prescribe the formulation best suited to a patient's needs and circumstances.

Anticipated tradeoffs are another important consideration for payers when evaluating fixed-dose SC formulations against weight-based IV administration. In a simulation analysis evaluating IV and SC rituximab formulations for non-Hodgkin lymphoma treatment in the US, the use of SC rituximab saved time compared to reference IV rituximab (ref-RITUX) and biosimilar IV rituximab (biosim-RITUX), and was generally cheaper 
than ref-RITUX (except for small-sized patients receiving a rapid infusion of the reference therapy). ${ }^{36}$ However, SC rituximab was more costly versus biosimRITUX in small- and average-sized patients (at all levels of biosimilar discount) as well as large-sized patients (at discounts ranging from $24 \%$ to $25 \%$ ). ${ }^{36}$ In a UK budget impact model assessing the cost of adoption of IV biosim-RITUX and IV biosimilar trastuzumab, the higher administration costs of the IV biosimilars versus SC were offset by lower acquisition costs, resulting in a lower total cost for IV biosimilars. ${ }^{37}$

Despite the benefits of SC administration to patients and the healthcare system, its wider adoption may be hampered by prevailing health insurance policies and physician reimbursement models. For instance, it has been suggested that higher out-of-pocket costs for selfinjectable medications for rheumatoid arthritis may result in stronger patient preference for infusion biologics. ${ }^{38}$ In the case of physicians, the buy-and-bill model incentivizes them to provide infusion treatments since payers can be billed for the cost of IV drugs administered in a clinical setting. Reimbursement for IV drugs generates considerable revenue, especially for oncology practices. ${ }^{39}$ An analysis of Medicare data from 2006 to 2009 assessed the factors associated with receipt of a given biologic (IV-administered infliximab vs SCadministered etanercept or adalimumab) in patients with rheumatoid arthritis starting their first anti-tumor necrosis factor therapy. ${ }^{38}$ The analysis found that stronger physician preference for infused therapies was related to reimbursement and also associated with a greater likelihood of infliximab administration. When physician preference for IV infusions was compared across the lowest to the highest quartiles, the proportion of physicians billing for infusions was $34 \%, 59 \%, 80 \%$, and $88 \%$, respectively. ${ }^{38}$

The incentivization of IV administration may also work against step therapy policies instituted by payers. To limit the utilization of infusion biologics, some payers require failure of SC therapy prior to payment authorization for the IV formulation. ${ }^{40,41}$ These step therapy policies have notable limitations. Firstly, they have been shown to have an equivocal effect on drug utilization $^{40}$ and, potentially, a limited cost benefit. ${ }^{41}$ Furthermore, while step therapy policies may constrain a HCP's initial product formulation choice, they may have a modest impact on the proportion of patients ultimately receiving IV therapy. These policies may simply delay the patient receiving the provider's preferred formulation, sometimes resulting in suboptimal care and additional expense. ${ }^{40}$ To improve patient outcomes and experience while reducing costs, it is imperative that efforts are made to address the misalignment of patient, physician, and payer interests concerning IV and $\mathrm{SC}$ formulations.

\section{Conclusions}

In making decisions about IV and SC therapies, payers must balance multiple considerations, including ease of coding and management, cost implications, patient and provider preference, and the aforementioned countervailing factors. Each drug should be evaluated based on the comparative merits of its IV and SC formulations, with patient well-being as the premier concern. The unintended effects of policies (eg, step therapy, buy-and-bill) impacting SC versus IV treatment choice should be acknowledged and addressed such that patients have access to treatments that best suit their needs and circumstances. Additionally, the specific features and attributes of drug delivery methods and technologies should be considered, recognizing, for instance, that not all SC delivery devices are created alike, and may therefore present distinct value propositions to different healthcare stakeholders. Future research including systematic literature reviews could be conducted to further elucidate the nuanced distinctions between these formulations across various products.

\section{Abbreviations}

Biosim-RITUX, biosimilar IV rituximab; COVID-19, coronavirus disease 2019; FDA, Food and Drug Administration; HCP, healthcare professional; HCPCS, Healthcare Common Procedure Coding System; IV, intravenous; IVIG, intravenous immunoglobulin; NDC, National Drug Code; PBM, pharmacy benefit management; ref-RITUX, reference IV rituximab; SC, subcutaneous; SCIG, subcutaneous immunoglobulin.

\section{Acknowledgments}

The author would like to thank J. Russell Teagarden, $\mathrm{DMH}, \mathrm{MA}$ for his advice in conceptualizing some of the payer considerations. Medical writing support, including assisting the author with the development of the manuscript drafts and incorporation of comments, was provided by Grace Jeong, PhD and Akua Adu-Boahene, MD, MPH of Alphabet Health (New York, NY), supported by 
Halozyme Therapeutics, according to Good Publication Practice guidelines (https://www.ismpp.org/gpp3).

\section{Funding}

Medical writing support was supported by Halozyme Therapeutics. The author received no honoraria related to the development of this publication.

\section{Disclosure}

Dr. Epstein currently serves on the Boards of Directors of Illumina, Fate Therapeutics, and Veracyte. He is Chairman of the Board of Decipher Biosciences. He has consulted with many life science companies including Halozyme, Merck, Boehringer Ingelheim, Astra Zeneca, Sanofi, G1 Therapeutics, Taiho, Radius Health, and ICTI. He reports personal fees from Halozyme, personal fees from G1 Therapeutics, personal fees from Taiho Oncology, personal fees from Merck, personal fees from Radius Health, personal fees from ICTI, personal fees from Diplomat Pharmacy, outside the submitted work.

\section{References}

1. National Home Infusion Association (NHIA). NHIA Home and Specialty Infusion Industry Recommendations for Providing Services During the COVID-19 Outbreak; 2019. Available from: https://www.nhia.org/covid-19_guidance/. Accessed 20 August 2020.

2. Cinar P, Kubal T, Freifeld A, et al. Safety at the Time of the COVID-19 Pandemic: how to Keep Our Oncology Patients and Healthcare Workers Safe. J National Compreh Cancer Net. 2020;18 (5):504.

3. Pharmaprojects. Pharma R\&D Annual Review 2019; 2019. Available from: https://pharmaintelligence.informa.com/ /media/informa-shopwindow/pharma/2019/files/whitepapers/pharma-rd-review-2019whitepaper.pdf. Accessed 27 October 2020.

4. Mikulic M Worldwide pipeline drugs by delivery route share 2019; 2019. Available from: https://www.statista.com/statistics/791731/ share-of-pipeline-drugs-worldwide-by-delivery-route/. Accessed 2 March 2020.

5. Schumock GT, Stubbings J, Hoffman JM, et al. National trends in prescription drug expenditures and projections for 2019. Am J Health Sys Pharmacy. 2019;76(15):1105-1121. doi:10.1093/ajhp/zxz109

6. Sanchez-Felix M, Burke M, Chen HH, Patterson C, Mittal S. Predicting bioavailability of monoclonal antibodies after subcutaneous administration: open innovation challenge. Adv Drug Deliv Rev. 2020;167:66-77. doi:10.1016/j.addr.2020.05.009

7. Johnson B. NDC Billing for Infusion and Injectable Medications. Available from: https://weinfuse.com/ndc-billing-for-infusion-andinjectable-medications/. Accessed 10 April 2020.

8. Faust S Pharmacy vs Medical Benefit. Available from: https://www. pharmacist.com/pharmacy-vs-medical-benefit. Accessed 27 October 2020.

9. Johnson B. Billing Infusion Medications. Available from: https://wein fuse.com/billing-infusion-medications/. Accessed 27 October 2020.

10. Burrows SH, Akinbobuyi O, Rule S, Crosbie N. Subcutaneous rituximab can be safely administered without pre-medication. $\mathrm{Br}$ J Haematol. 2018;181(6):836-837. doi:10.1111/bjh.14703
11. Human Genome Sciences, Inc. BENLYSTA (Belimumab) [Package Insert]. Rockville, MD: Human Genome Sciences, Inc., (a subsidiary of GlaxoSmithKline); 2020.

12. Schmier J, Ogden K, Nickman N, et al. Costs of Providing Infusion Therapy for Rheumatoid Arthritis in a Hospital-based Infusion Center Setting. Clin Ther. 2017;39(8):1600-1617. doi:10.1016/j.clinthera.20 17.06.007

13. Wu N, Bhurke S, Shah N, Harrison DJ. Application of a validated algorithm to estimate the effectiveness and cost of biologics for rheumatoid arthritis in the US pharmacy benefit manager context. Clinicoecon Outcomes Res. 2015;7:257-266. doi:10.2147/CEOR.S83932

14. Hansen RN, Wallick CJ, Sullivan SD. Budget Impact of the Introduction of Subcutaneous Rituximab to U.S. Health Plans. Value in Health. 2018;21:S22-S23. doi:10.1016/j.jval.2018.04.137

15. Tetteh EK, Morris S. Evaluating the administration costs of biologic drugs: development of a cost algorithm. Health Econ Rev. 2014;4 (1):26. doi:10.1186/s13561-014-0026-2

16. Anderson KC, Landgren O, Arend RC, Chou J, Jacobs IA. Humanistic and economic impact of subcutaneous versus intravenous administration of oncology biologics. Future Oncol. 2019;15 (28):3267-3281. doi:10.2217/fon-2019-0368

17. Plommet N, Pau D, Tehard B. Economic Evaluation and Added Value for Stakeholders of Switching From Rituximab Intravenous Injection to Rituximab Subcutaneous Injection In France. Value in Health. 2015;18(7):A664. doi:10.1016/j.jval.20 15.09.2418

18. Rule S, Collins GP, Samanta K. Subcutaneous vs intravenous rituximab in patients with non-Hodgkin lymphoma: a time and motion study in the United Kingdom. J Med Econ. 2014;17(7):459-468. doi:10.3111/13696998.2014.914033

19. Olofsson S, Norrlid H, Karlsson E, Wilking U, Ragnarson Tennvall G. Societal cost of subcutaneous and intravenous trastuzumab for HER2-positive breast cancer - An observational study prospectively recording resource utilization in a Swedish healthcare setting. Breast. 2016;29:140-146. doi:10.1016/j.breast.20 16.07.008

20. Iuliano F, Iuliano E, Rossi M. Assisted Administration of Subcutaneous Rituximab to the Patient's Home. Significant Reduction of Indirect Costs Incurred By Care Recipients and Unpaid Caregivers Together with a Total Adherence to the Treatment Schedule and Same Safety. Blood. 2018;132(Supplement 1):5869. doi:10.1182/blood-2018-99-118756

21. Edo Solsona MD, Monte Boquet E, Casanova Estruch B, Poveda Andrés JL. Impact of adherence on subcutaneous interferon beta-1a effectiveness administered by Rebismart $\left({ }^{\circledR}\right)$ in patients with multiple sclerosis. Patient Prefer Adherence. 2017;11:415-421. doi:10.2147/ PPA.S127508

22. Bittner B, Schmit Chiesi C, Kharawala S, Kaur G, Schmidt J. Connected drug delivery devices to complement drug treatments: potential to facilitate disease management in home setting. Med Devices. 2019;12:101-127. doi:10.2147/MDER.S198943

23. Centers for Medicare \& Medicaid Services (CMS). Physicians and Other Clinicians: CMS Flexibilities to Fight COVID-19. Available from: https://www.cms.gov/files/document/covid-19-physicians-andpractitioners.pdf. Accessed 8 October 2020.

24. Lugaresi A, Rottoli MR, Patti F. Fostering adherence to injectable disease-modifying therapies in multiple sclerosis. Expert Rev Neurother. 2014;14(9):1029-1042. doi:10.1586/14737175.2014.945523

25. Bayas A. Improving adherence to injectable disease-modifying drugs in multiple sclerosis. Expert Opin Drug Deliv. 2013;10(3):285-287. doi:10.1517/17425247.2013.763793

26. Chari A, Goldschmidt H, San-Miguel J, et al. Subcutaneous (SC) Daratumumab (DARA) in Combination With Standard Multiple Myeloma (MM) Treatment Regimens: an Open-label, Multicenter Phase 2 Study (PLEIADES). Clin Lymphoma Myeloma Leuk. 2019;19(10):e16-e17. doi:10.1016/j.clml.2019.09.023 
27. Usmani SZ, Nahi H, Mateos MV, et al. Subcutaneous delivery of daratumumab in relapsed or refractory multiple myeloma. Blood. 2019;134(8):668-677. doi:10.1182/blood.2019000667

28. Mateos M-V, Nahi H, Legiec W, et al. Efficacy and safety of the randomized, open-label, non-inferiority, Phase 3 study of subcutaneous (SC) versus intravenous (IV) daratumumab (DARA) administration in patients (pts) with relapsed or refractory multiple myeloma (RRMM): COLUMBA. J Clin Oncol. 2019;37(15_suppl):8005. doi:10.1200/JCO.2019.37.15 suppl.8005

29. Kim HJ, Yun J, Kim HJ, et al. Safety and effectiveness of central venous catheterization in patients with cancer: prospective observational study. J Korean Med Sci. 2010;25(12):1748-1753. doi:10.3346/jkms.2010.25.12.1748

30. Dashiell-Aje E, Harding G, Pascoe K, DeVries J, Berry P, Ramachandran S. Patient Evaluation of Satisfaction and Outcomes with an Autoinjector for Self-Administration of Subcutaneous Belimumab in Patients with Systemic Lupus Erythematosus. Patient. 2018;11(1):119-129. doi:10.1007/s40271-017-0276-2

31. Stoner KL, Harder H, Fallowfield LJ, Jenkins VA. Intravenous versus Subcutaneous Drug Administration. Which Do Patients Prefer? A Systematic Review. Patient. 2014. doi:10.1007/s40271-014-0075-y

32. Collins DS, Sanchez-Felix M, Badkar AV, Mrsny R. Accelerating the development of novel technologies and tools for the subcutaneous delivery of biotherapeutics. J Control Release. 2020;321:475-482. doi:10.1016/j.jconrel.2020.02.036

33. Vaughan LJ. Managing cost of care and healthcare utilization in patients using immunoglobulin agents. Am J Manag Care. 2019;25 (6 Suppl):S105-S111.

34. Ness S. Differentiating characteristics and evaluating intravenous and subcutaneous immunoglobulin. Am J Manag Care. 2019;25(6 Suppl): S98-S104.
35. Rhodes RT, McFalls K A Comparative Look at IVIG and SCIG. Available from: http://www.nufactor.com/healthcare/resources/ig/articles/ar-a-com parative-look-at-ivig-and-scig.html. Accessed September 9, 2019.

36. McBride A, Balu S, Campbell K, MacDonald K, Abraham I. Subcutaneous Versus Intravenous Rituximab in Non-Hodgkin Lymphoma Treated with R-CHOP: economic Modeling for the US. Blood. 2018;132(Supplement 1):4776. doi:10.1182/blood-2018-99113283

37. Jang Y, Byrne A, Toron F, Yoon S Budget Impact Analysis of Intravenous Biosimilars Compared With Intravenous Originators and Subcutaneous Products. Presented at 2018 International Society for Pharmacoeconomics and Outcomes Research (ISPOR) Europe Conference. November 12 2018. Paper presented at: Presented at 2018 International Society for Pharmacoeconomics and Outcomes Research (ISPOR) Europe Conference. 2018; Barcelona, Spain.

38. Zhang J, Xie F, Delzell E, et al. Trends in the use of biologic agents among rheumatoid arthritis patients enrolled in the US medicare program. Arthritis Care Res. 2013;65(11):1743-1751. doi:10.1002/acr.22055

39. Pakizegee M, Stefanacci RG. Clinical pathways and policy to guard against misaligned incentives. J Clin Pathways. 2020;6(1):34-36.

40. Kozma CM, Ingham M, Paris A, Ellis L. Effectiveness of step therapy policies for specialty pharmaceuticals in immune disorders. J Med Econ. 2015;18(8):646-653. doi:10.3111/13696998.2015.1035278

41. Ingham M, Paris A, Ellis L, Kozma C. One approach to evaluating step therapy policies for immune disorder specialty pharmaceuticals. Ann Rheum Dis. 2013;72:A564-A564. doi:10.1136/annrheumdis-2013-eular. 1685
ClinicoEconomics and Outcomes Research

\section{Publish your work in this journal}

ClinicoEconomics and Outcomes Research is an international, peerreviewed open-access journal focusing on Health Technology Assessment, Pharmacoeconomics and Outcomes Research in the areas of diagnosis, medical devices, and clinical, surgical and pharmacological intervention. The economic impact of health policy and health systems

\section{Dovepress}

organization also constitute important areas of coverage. The manuscript management system is completely online and includes a very quick and fair peer-review system, which is all easy to use. Visit $\mathrm{http}: / / \mathrm{www}$.dovepress.com/testimonials.php to read real quotes from published authors.

Submit your manuscript here: https://www.dovepress.com/clinicoeconomics-and-outcomes-research-journal 